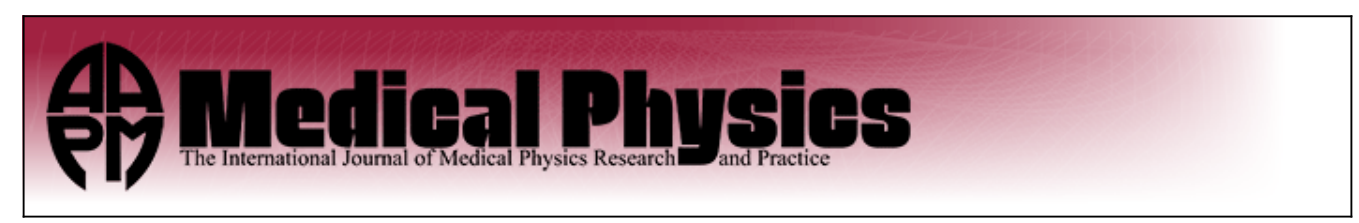

\title{
Collimator design for a multipinhole brain SPECT insert for MRI
}

Karen Van Audenhaege, Roel Van Holen, Christian Vanhove, and Stefaan Vandenberghe

Citation: Medical Physics 42, 6679 (2015); doi: 10.1118/1.4934371

View online: http://dx.doi.org/10.1118/1.4934371

View Table of Contents: http://scitation.aip.org/content/aapm/journal/medphys/42/11?ver=pdfcov

Published by the American Association of Physicists in Medicine

\section{Articles you may be interested in}

Multipinhole SPECT helical scan parameters and imaging volume

Med. Phys. 42, 6599 (2015); 10.1118/1.4933421

Segmented slant hole collimator for stationary cardiac SPECT: Monte Carlo simulations

Med. Phys. 42, 5426 (2015); 10.1118/1.4928484

Multipinhole collimator with 20 apertures for a brain SPECT application

Med. Phys. 41, 112501 (2014); 10.1118/1.4897567

Analytical, experimental, and Monte Carlo system response matrix for pinhole SPECT reconstruction

Med. Phys. 41, 032501 (2014); 10.1118/1.4866380

Performance of a high-sensitivity dedicated cardiac SPECT scanner for striatal uptake quantification in the brain based on analysis of projection data

Med. Phys. 40, 042504 (2013); 10.1118/1.4794488

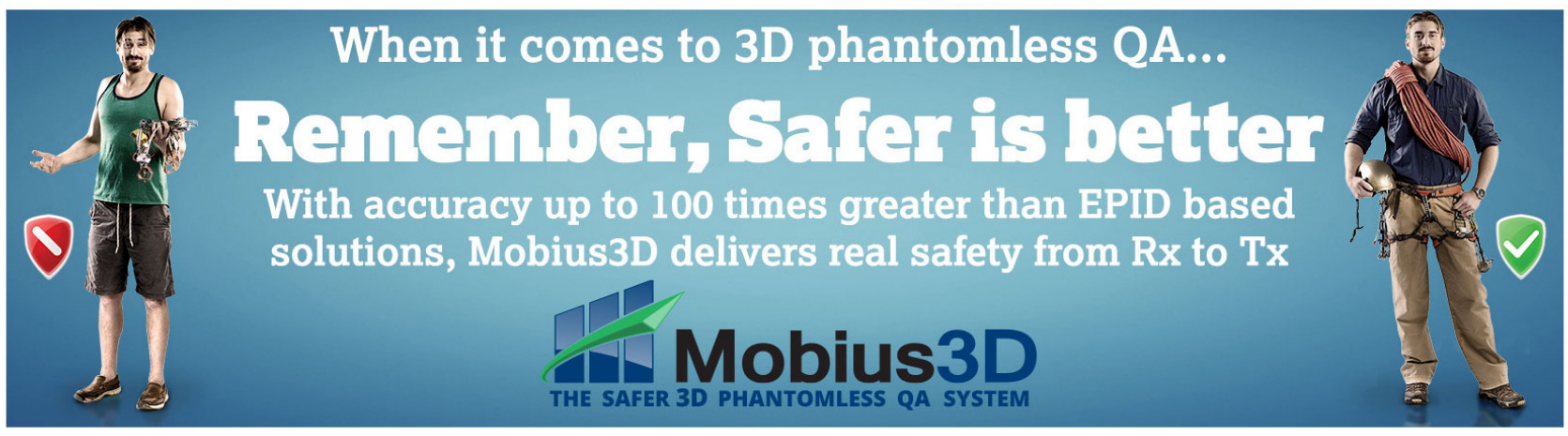




\title{
Collimator design for a multipinhole brain SPECT insert for MRI
}

\author{
Karen Van Audenhaege, Roel Van Holen, ${ }^{\text {a) }}$ Christian Vanhove, and Stefaan Vandenberghe \\ Department of Electronics and Information Systems, Ghent University-iMinds Medical IT, MEDISIP-IBiTech, \\ De Pintelaan 185 block B/5, Ghent B-9000, Belgium
}

(Received 13 June 2015; revised 29 September 2015; accepted for publication 10 October 2015; published 26 October 2015)

Purpose: Brain single photon emission computed tomography (SPECT) imaging is an important
clinical tool, with unique tracers for studying neurological diseases. Nowadays, most commercial
SPECT systems are combined with x-ray computed tomography (CT) in so-called SPECT/CT
systems to obtain an anatomical background for the functional information. However, while CT
images have a high spatial resolution, they have a low soft-tissue contrast, which is an important
disadvantage for brain imaging. Magnetic resonance imaging (MRI), on the other hand, has a very
high soft-tissue contrast and does not involve extra ionizing radiation. Therefore, the authors designed
a brain SPECT insert that can operate inside a clinical MRI. Methods: The authors designed and simulated a compact stationary multipinhole SPECT insert based on digital silicon photomultiplier detector modules, which have shown to be MR-compatible and have an excellent intrinsic resolution $(0.5 \mathrm{~mm})$ when combined with a monolithic $2 \mathrm{~mm}$ thick LYSO crystal. First, the authors optimized the different parameters of the SPECT system to maximize sensitivity for a given target resolution of $7.2 \mathrm{~mm}$ in the center of the field-of-view, given the spatial constraints of the MR system. Second, the authors performed noiseless simulations of two multipinhole configurations to evaluate sampling and reconstructed resolution. Finally, the authors performed Monte Carlo simulations and compared the SPECT insert with a clinical system with ultrahigh-resolution (UHR) fan beam collimators, based on contrast-to-noise ratio and a visual comparison of a Hoffman phantom with a $9 \mathrm{~mm}$ cold lesion.

Results: The optimization resulted in a stationary multipinhole system with a collimator radius of $150.2 \mathrm{~mm}$ and a detector radius of $172.67 \mathrm{~mm}$, which corresponds to four rings of 34 diSPM detector modules. This allows the authors to include eight rings of 24 pinholes, which results in a system volume sensitivity of $395 \mathrm{cps} / \mathrm{MBq}$. Noiseless simulations show sufficient axial sampling (in a Defrise phantom) and a reconstructed resolution of $5.0 \mathrm{~mm}$ (in a cold-rod phantom). The authors compared the 24-pinhole setup with a 34-pinhole system (with the same detector radius but a collimator radius of $156.63 \mathrm{~mm}$ ) and found that 34 pinholes result in better uniformity but a worse reconstruction of the cold-rod phantom. The authors also compared the 24-pinhole system with a clinical triple-head UHR fan beam system based on contrast-to-noise ratio and found that the 24-pinhole setup performs better for the $6 \mathrm{~mm}$ hot and the $16 \mathrm{~mm}$ cold lesions and worse for the 8 and $10 \mathrm{~mm}$ hot lesions. Finally, the authors reconstructed noisy projection data of a Hoffman phantom with a $9 \mathrm{~mm}$ cold lesion and found that the lesion was slightly better visible on the multipinhole image compared to the fan beam image.

Conclusions: The authors have optimized a stationary multipinhole SPECT insert for MRI and showed the feasibility of doing brain SPECT imaging inside a MRI with an image quality similar to the best clinical SPECT systems available. (C) 2015 American Association of Physicists in Medicine. [http://dx.doi.org/10.1118/1.4934371]

Key words: SPECT, collimator, production, single photon emission computed tomography, optimization

\section{INTRODUCTION}

Combining single photon emission computed tomography (SPECT) with magnetic resonance imaging (MRI) for additional anatomical information has two important advantages compared to the combination with $\mathrm{x}$-ray computed tomography $(\mathrm{CT})$, which is the current standard. First, MRI provides superior soft-tissue contrast to $\mathrm{CT}$, which is important in brain imaging as the brain consists mainly of cerebro-spinal fluid and white and gray matter. And second, MRI does not involve any radiation dose like CT. In clinical settings, SPECT and MR images are often combined using software fusion but if SPECT and MRI could be fully integrated, it would increase patient throughput, reduce total patient examination time, and eliminate coregistration errors. It would also enable the possibility of simultaneous dynamic SPECT and MRI, which is of interest in research.

Integrating SPECT with MRI is challenging for a number of reasons. First, the attenuation maps that are used during SPECT reconstruction and that are normally derived from CT 
cannot directly be obtained from the MRI and an intermediate step is needed to obtain the attenuation map. ${ }^{1}$ Techniques to derive the attenuation map from MRI are based on segmentation and atlas registration, which has some drawbacks related to interpatient variability. Second, the collimator should not contain any ferromagnetic materials in order not to disturb the $B_{0}$ field of the MRI. It should ideally be made of a material with a magnetic susceptibility close to human tissue. Third, the detector modules need to be able to operate in the strong magnetic field. Currently, most clinical SPECT detectors are based on photomultiplier tubes (PMTs) that cannot operate in a magnetic field and give rise to $B_{0}$ magnetic field distortions. Fourth, the SPECT system should be stationary in order not to perturb the magnetic field. A stationary system does not require any translational or rotational steps and is therefore not only more stable but also makes it possible to perform dynamic studies. However, most current SPECT systems rely on rotation to acquire images at different projection angles and it is challenging to obtain sufficient angular sampling with a stationary system. Finally, the MR gradients cause eddy currents in the collimator material (typically lead) which can perturb the MR gradient field and should be minimized for successfully integrating SPECT and MRI. ${ }^{2}$ Finally, the SPECT system should fit within the bore of a clinical MRI scanner with a diameter of $70 \mathrm{~cm}$. However, current clinical systems are bulky dual- or triple-head gamma cameras equipped with fan beam or parallel beam collimators.

Some of the above challenges have already been addressed during the development of MRI-compatible positron emission tomography (PET). For example, the MRI-compatibility of different gamma shielding materials has been investigated for PET, ${ }^{3}$ but the results can also be used for SPECT. Also useful are the MRI-compatibility tests of different PET detector modules. The first MRI-compatible PET systems were based on avalanche photodiodes (APDs). ${ }^{4}$ More recently, most MRIcompatible PET designs are based on silicon photomultiplier (SiPM) detector modules ${ }^{5,6}$ or digital silicon photomultiplier (dSiPM) detector modules. ${ }^{7}$ We refer the interested reader to Ref. 8 for an extensive review of PET/MRI. Additional challenges of SPECT/MRI (compared to PET/MRI) are the space required for the collimator and potential eddy current interferences with MRI.

While the developments in PET/MRI resulted in both clinical $^{9-12}$ and preclinical systems, ${ }^{7,13-16}$ research on SPECT/ MRI is still mainly focussed on small-animal systems. The earliest SPECT/MRI systems were based on cadmium-zinctelluride (CZT) detector modules. For example, the MRSPECT was developed in 2010 as a proof of concept and is based on a parallel hole collimator and a whole body $4 \mathrm{~T}$ MRI system and requires a rotation of the object. ${ }^{17} \mathrm{~A}$ fully stationary system was also developed using CZT detector modules, for a $12-\mathrm{cm}$ bore preclinical MRI system. ${ }^{18-20}$ Similarly, Meng et al. developed a SPECT insert for a $22-\mathrm{cm}$ bore 4.7 T MRI. ${ }^{21,22}$ More recently, the MRC-SPECT (Ref. 23) has been built. This ultrahigh-resolution (UHR) system is based on cadmium-telluride (CdTe) detector modules and multipinhole collimators and is also stationary. It fits within a preclinical MRI system. Another small-animal system is still under development for the INSERT project. ${ }^{24}$ It is designed to fit within a preclinical 7 or 9.4 T MRI system with a $20 \mathrm{~cm}$ bore diameter and is based on SiPMs and multipinhole collimators and is stationary. A human brain SPECT/MRI is also planned within the same project. ${ }^{24}$ It is designed to fit within a $59-\mathrm{cm}$ clinical 3 T MRI system and is also stationary and based on SiPMs and multipinhole collimators.

All these systems are still in research phase and no truly integrated SPECT/MRI systems are yet commercially available. At this moment, Mediso Medical Imaging Systems ${ }^{25}$ is the only company offering a preclinical $1 \mathrm{~T}$ sequential SPECT/MRI system (nanoScan SPECT/MRI).

In this study, we design a SPECT insert for MRI based on a multipinhole collimator and $\mathrm{dSiPM}$ detector modules, which have shown to be MR-compatible ${ }^{7}$ and have an excellent intrinsic resolution $(0.5 \mathrm{~mm})$ and an energy resolution of $20 \%$ (at $140 \mathrm{keV}$ ) when combined with a monolithic $2 \mathrm{~mm}$ thick LYSO crystal. ${ }^{26}$ An excellent detector resolution makes it possible to design a multipinhole system with minification, ${ }^{27}$ as opposed to traditional multipinhole systems, which use magnification to obtain a good spatial resolution by placing the detector relatively far from the pinholes (phs) [see Eq. (1)]. Minification, on the other hand, allows the system to be compact, which is needed to fit inside a clinical MRI and makes it possible to obtain more angular projections simultaneously ${ }^{28}$ so that we can achieve complete transverse sampling with a full-ring multipinhole collimator without rotating the camera.

We will first describe the general design of the collimator and detector ring and then describe the optimization process used to maximize the volume sensitivity for a target resolution of $7.2 \mathrm{~mm}$ in the center of the brain. Finally, we will simulate and reconstruct both noiseless and noisy phantom data to assess reconstructed spatial resolution, angular sampling, and image quality. We will also compare the system to a clinical system with the same resolution: a triple-head SPECT camera equipped with ultrahigh-resolution fan beam collimators (UHR FAN).

\section{MATERIALS AND METHODS}

\section{A. Multipinhole system design and optimization}

Figure 1 illustrates the general design of the SPECT insert. It is inspired by existing small-animal SPECT/MRI inserts ${ }^{23,24}$ and consists of a detector ring and a cylindrical multipinhole collimator. The insert fits within the MR bore and is positioned between the gradient coils and the Tx/Rx coil, which should be close to the patient's head for optimal performance.

\section{A.1. Detector design}

The detector geometry is cylindrical, based on dSiPM detector modules of $32 \times 32 \mathrm{~mm}^{2}$ [Fig. 2(c)]. The detector modules have shown to be MR-compatible ${ }^{7}$ and have an excellent intrinsic resolution $(0.5 \mathrm{~mm})$ and an energy resolution of $20 \%$ (at $140 \mathrm{keV}$ ) when combined with a monolithic $2 \mathrm{~mm}$ thick LYSO crystal. ${ }^{26}$ Excellent spatial resolution makes it possible 


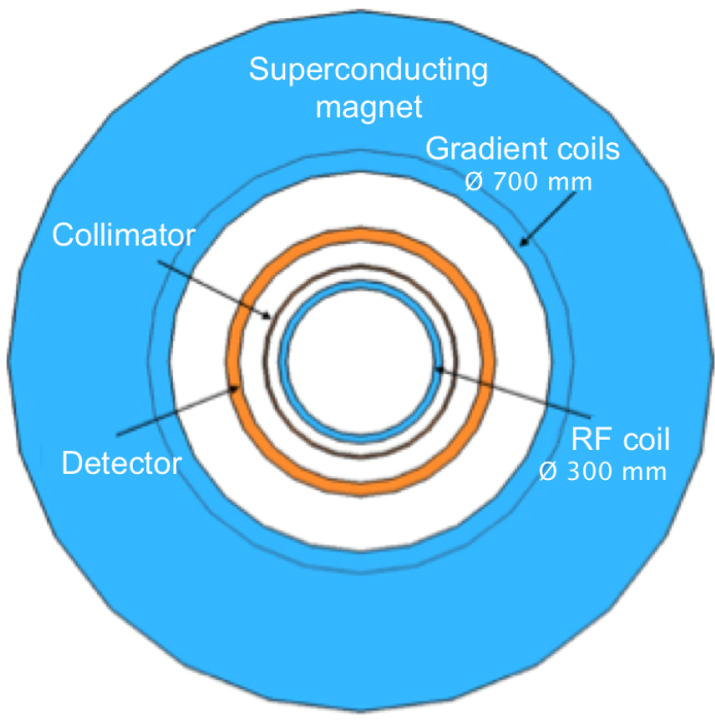

Fig. 1. Configuration of the SPECT system (collimator and detector) inside the MRI, between the RF coil and the gradient coils. The RF coil is both a transmitter and receiver $(\mathrm{Tx} / \mathrm{Rx})$ and its dimensions are based on an eight channel Rx/Tx head coil.

to design a multipinhole system with minification and acquire more angular projections simultaneously (Fig. 3), which is needed to obtain sufficient angular sampling without rotating the system. The detector modules have a size of $32 \times 32 \mathrm{~mm}^{2}$, but only $30 \times 30 \mathrm{~mm}^{2}$ can be used for correctly positioning the detected events. This causes gaps in the detector ring [Fig. 2(c)], which could introduce artifacts. To investigate this, we also modeled the gaps in the simulation study. The radius of the detector ring is optimized in Sec. 2.A.3.

\section{A.2. Collimator design}

Figures 2(a), 2(b) and 4 show the collimator in more detail. The pinholes are positioned on rings that are separated by slats to avoid overlap of pinhole projections in the axial direction.
We chose to use eight rings as a compromise between axial sampling and loss of sensitivity due to the axial slats. Simulations will show that eight rings are sufficient for this design. The rings are rotated relative to one another to improve angular sampling.

A ring-shaped collimator is difficult to produce with traditional manufacturing techniques but can be built with additive manufacturing using laser sintering of pure tungsten powder. In contrast to the tungsten alloys that are used in traditional manufacturing techniques, tungsten powder does not contain any ferromagnetic materials and is therefore interesting for building MR-compatible SPECT system..$^{29,30}$

\section{A.3. System optimization}

It is our goal to optimize the SPECT insert to achieve the highest possible sensitivity while the total detector surface area is fixed and the system spatial resolution matches the current state-of-the-art. We chose a detector surface area of $1393 \mathrm{~cm}^{2}$ (136 dSiPMs), which corresponds to the area that is maximally irradiated when a human brain is scanned on the clinical triplehead PRISM XP 3000 with a fan beam collimator, and we chose a spatial resolution of $7.2 \mathrm{~mm}$ in the center of the FOV, which is comparable to the resolution of the UHR fan beam system. We assume the FOV to be cylindrical with a diameter of $220 \mathrm{~mm}$ and an axial length of $124 \mathrm{~mm}$ which is representative for an adult human brain. ${ }^{31}$ We included six design parameters in the optimization study: the detector radius $D$, the collimator radius $c$, the pinhole aperture diameter $d$, the pinhole opening angle $\alpha$, the number of pinholes per ring $N_{p}$, and the detector length $L_{D}$. Other parameters are collimator material thickness $t$, pinhole position, pinhole tilt, and detector length. To simplify the collimator design, all the pinholes have the same aperture diameter, opening angle, and tilt. These pinholes all view the complete transverse FOV without allowing overlap of the pinhole projections, are not tilted in the axial direction, and all focus on the center in the transverse direction. a)

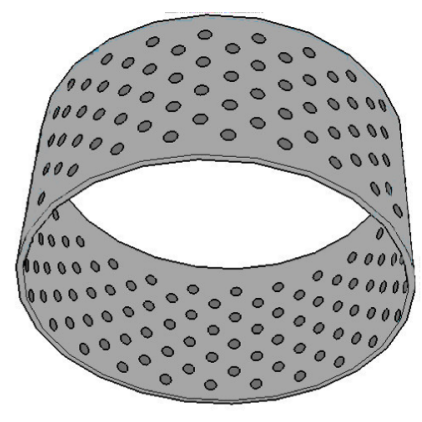

b)

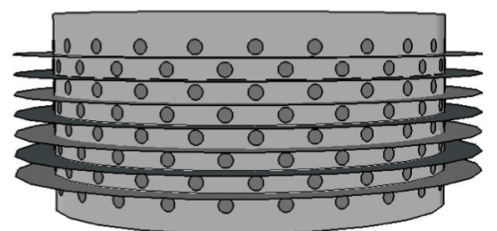

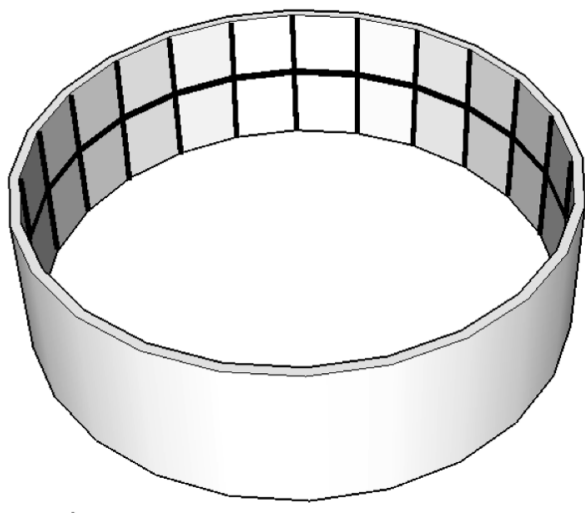

c)

Fig. 2. (a) Detail of the collimator, (b) collimator with slats to separate projections in axial direction, (c) detector ring composed of $32 \times 32 \mathrm{~mm}^{2}$ detector components with a small gap between them. 


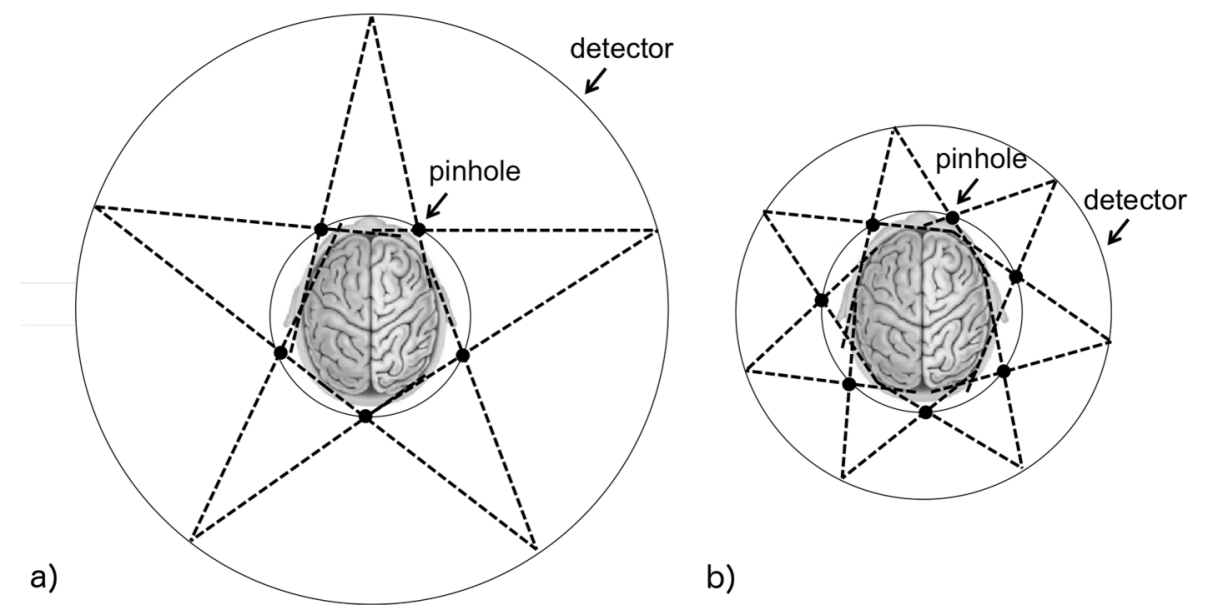

Fig. 3. (a) Magnifying system: Traditionally, the detector is placed relatively far away from the pinholes which results in magnified projections. (b) Minifying system: The collimator-detector distance is smaller than the collimator radius, which results in minifying projections and allows to acquire more angular projections simultaneously.

The collimator thickness is $5 \mathrm{~mm}$. The axial length of the detector depends on $D$ and the fixed detector surface area.

We used an analytical optimization process that was previously described in Ref. 32 and can be summarized as follows:

- Loop over all values of $D$ (between 150 and $350 \mathrm{~mm}$, respectively, the outer radius of an eight channel $\mathrm{Tx} / \mathrm{Rx}$ head coil and the gradient inner diameter of a clinical MRI).

- Loop over all values of $c$ (between $150 \mathrm{~mm}$ and $D$ ).

- For each combination of $c$ and $D$ :

- Determine the number of pinholes per ring $\left(N_{p}\right)$ and their opening angle $(\alpha)$ using the geometrical relationship that follows from the fact that all pinholes view the complete transverse FOV without allowing overlap of the pinhole projections.

- Derive the pinhole aperture diameter $d$ needed to achieve the target resolution in the center of the FOV, using the formulas presented by Refs. 33 and 34,

$R_{\mathrm{CFOV}}=\sqrt{\frac{R_{i}^{2}}{m^{2}}+\left(d_{\mathrm{Reff}}\left(1+\frac{1}{m}\right)\right)^{2}}$.

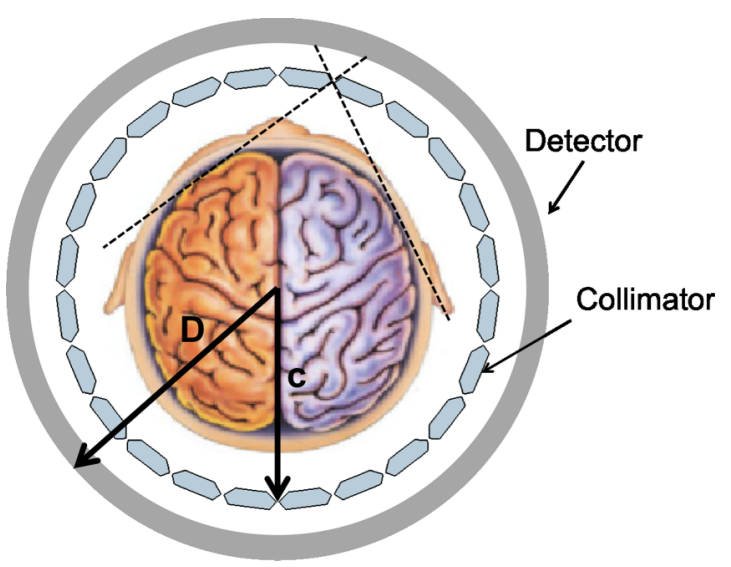

FIG. 4. A transverse slice of the SPECT system with collimator radius $c$ and detector radius $D$
$R_{i}$ is the intrinsic resolution of the detector, which is assumed to be $0.5 \mathrm{~mm}, d_{\text {Reff }}$ is the resolution effective diameter (corrected for penetration), $R_{\mathrm{CFOV}}$ is the target resolution $(7.2 \mathrm{~mm})$, and $m$ is the magnification of the system and is described by

$m=\frac{D-c}{c}$.

The resolution effective diameter $d_{\text {Reff }}$ is described by two equations, ${ }^{34}$ which reduces to the same equation for a point source in the center of the FOV,

$d_{\text {Reff }}=d+\frac{\ln 2}{\mu} \tan \frac{\alpha}{2}$.

Equations (1)-(3) are then combined and rearranged to explicitly show $d$,

$d=\sqrt{\frac{(D-c)^{2} \cdot R_{\mathrm{CFOV}}^{2}-c^{2} R_{i}^{2}}{D^{2}}}-\frac{\ln 2}{\mu} \tan \frac{\alpha}{2}$.

- Determine the axial length of the detector ring by dividing the fixed detector surface area $\left(1393 \mathrm{~cm}^{2}\right)$ by the circumference of the detector ring $(2 \pi D)$.

- Calculate volume sensitivity.

- Determine the volume sensitivity for each combination of $c$ and $D$ and find the maximum.

To simplify the optimization, we approximated the detector to a perfect ring (instead of a polygon composed of flat detector modules).

\section{B. Phantom simulations}

After optimizing the design, we simulated and reconstructed phantom data to evaluate system performance. First, we evaluated spatial resolution and sampling sufficiency in two different multipinhole designs (one with more pinholes than the other). Therefore, we simulated and reconstructed noiseless projection data of three phantoms, namely, a coldrod phantom [Fig. 6(a)], a Defrise phantom [Fig. 7(a)], and a contrast phantom [Fig. 6(d)]. We also compared the 
multipinhole SPECT insert with a clinical triple-head fan beam system. Therefore, we simulated noisy data of the contrast phantom and evaluated the contrast-to-noise ratio (CNR) in both systems. Finally, we also simulated and reconstructed noisy projection data of a Hoffman phantom [Fig. 10(a)] to represent a more clinically relevant situation and compared the images visually.

\section{B.1. Noiseless simulations}

All noiseless projection data were simulated using a GPUbased pixel-driven forward projector ${ }^{35}$ that was previously developed in our research group ${ }^{28,36}$ and is based on the principle of ray-tracing, using 456 rays to model the finite dimension of the pinhole opening. An effective pinhole aperture diameter ${ }^{34}$ was used to model pinhole penetration. The phantoms were modeled using a grid with $240 \times 240 \times 240$ voxels of $1 \times 1 \times 1 \mathrm{~mm}^{3}$ and each detector was modeled using $300 \times 300$ pixels of $0.1 \times 0.1 \mathrm{~mm}^{2}$. This results in an active detector area of $300 \times 300 \mathrm{~mm}^{2}$ and $2 \mathrm{~mm}$ gaps between the detector modules. After the simulation, we applied a Gaussian blur of $0.5 \mathrm{~mm}$ full-width at half-maximum (FWHM) to the projection data to model the intrinsic resolution of the detector and then rebinned the projection data to a pixel size of 0.5 $\times 0.5 \mathrm{~mm}^{2}$. Attenuation was modeled analytically based on the assumption that the phantoms are made of polymethyl methacrylate (PMMA) and filled with water. No scatter was modeled.

\section{B.2. Noisy simulations}

Noisy simulations were performed using the Monte Carlo simulator GATE version $6.1 .{ }^{37}$ We simulated phantoms filled with $111 \mathrm{MBq}$ of Tc-99m and an acquisition time of $30 \mathrm{~min}$. The simulation incorporated the full-ring multipinhole collimator, the scintillator material (for modeling the stopping power), the detector's intrinsic spatial resolution, the detector's energy resolution, and the gaps. Again, the pixel size was rebinned to $0.5 \times 0.5 \mathrm{~mm}^{2}$ pixels after applying a Gaussian blur to model the intrinsic resolution of the detector. Scatter and attenuation were modeled based on the assumption that the phantoms are made of PMMA and filled with water. We performed the noisy simulations both for the multipinhole SPECT insert and for a clinical fan beam system.

\section{B.3. Phantoms}

All phantoms had a cylindrical shape with a diameter of $220 \mathrm{~mm}$ and a length of $124 \mathrm{~mm}$.

Cold-rod phantom. The cold-rod phantom was used to evaluate the reconstructed spatial resolution of the SPECT insert. The phantom contains six segments with rods with diameters of 10, 8, 6.5, 5, 4, and $2 \mathrm{~mm}$. The distance between the cold rods is twice their diameter [Fig. 6(a)].

Defrise phantom. A Defrise phantom was used to evaluate the axial sampling. It consists of $8 \mathrm{~mm}$-thick disks with a pitch of $8 \mathrm{~mm}$ [Fig. 7(a)].
Contrast phantom. The contrast phantom was used to evaluate contrast-to-noise as a measure of image quality. It has four hot rods (uptake ratio 7:1 and diameters of 6, 8,10, and $12 \mathrm{~mm}$ ) and two cold rods (diameters of 14 and $16 \mathrm{~mm}$ ) positioned on a circumferential ring of radius $54.5 \mathrm{~mm}$ [Fig. 6(d)].

Hoffman phantom. Finally, we performed noisy simulations of a Hoffman phantom modeled as a voxelized phantom with $1 \times 1 \times 1 \mathrm{~mm}^{3}$ voxels. The phantom has a spherical cold lesion with a diameter of $9 \mathrm{~mm}$ with zero activity [Fig. 10(a)]. The simulated uptake ratio between gray and white matter is $5: 1$ as in the physical phantom. ${ }^{38}$

\section{B.4. Clinical triple-head UHR fan beam system}

We compared the performance of the multipinhole SPECT insert with a PRISM 3000XP (a clinical triple-head SPECT system) equipped with UHR fan beam collimators. The PRISM has three rotating detector heads with $\mathrm{NaI}$ crystals and axial and transverse dimensions of, respectively, 242 and $406 \mathrm{~mm}$. The detector intrinsic spatial and energy resolutions are $3.0 \mathrm{~mm}$ and $10 \%$, respectively. The UHR fan beam collimator has a height of $34.9 \mathrm{~mm}$, a hole size of $1.4 \mathrm{~mm}$, and a focal length of $50 \mathrm{~cm}$, resulting in a system spatial resolution of $7.2 \mathrm{~mm}$ in the center of the FOV and a volume sensitivity of $3 \times 160 \mathrm{cps} / \mathrm{MBq}=480 \mathrm{cps} / \mathrm{MBq}$. The septal thickness is $0.15 \mathrm{~mm}$. We simulated an acquisition with 40 rotation steps, which results in 120 projection angles (as there are three detector heads).

\section{B.5. Image reconstruction}

The noisy and noiseless multipinhole projection data were reconstructed with maximum likelihood expectation maximization (MLEM). MLEM was implemented using a pixeldriven forward projector modeling the finite dimension of the pinhole opening using seven rays ${ }^{39}$ that cover the pinhole area with Gaussian quadrature weights and a voxel-driven back projector with one ray. Attenuation, sensitivity and spatial resolution are only modeled in the forward projector.

The fan beam projection data were first rebinned to parallel beam data and then reconstructed using MLEM. For every projection angle, the estimated image was rotated using a cubic spline rotator ${ }^{40}$ and then forward projected toward each pixel, while the back projector was voxel-driven. Resolution was modeled using a blurring operation in image space with a distance dependent FWHM. Sensitivity was modeled using a distance dependent weighting factor. We used the sensitivity and resolution formulas described by Moyer. ${ }^{41}$

All reconstructed images were represented within a 128 $\times 128 \times 128$ matrix with $1.875 \times 1.875 \times 1.875 \mathrm{~mm}^{3}$ voxels .

\section{B.6. Contrast-to noise}

We compared the fan beam system and the multipinhole insert quantitatively, based on contrast-to-noise ratio. Therefore, we used the contrast phantom as described in Sec. 2.B.3 and plotted the contrast recovery coefficient (CRC) at each 
image reconstruction iteration as a function of the coefficient of variation $(\mathrm{COV})$. The $\mathrm{CRC}$ and $\mathrm{COV}$ values are averaged over nine observations coming from nine $7.5-\mathrm{mm}$ slices of the contrast phantom, spaced $11.25 \mathrm{~mm}$ apart (center-to-center distance).

The CRC is defined as follows:

$$
\mathrm{CRC}(\%)=\frac{1}{N_{n}} \sum_{j=1}^{N_{n}}\left(\frac{\bar{L}_{j}-\bar{B}_{j}}{\bar{B}_{j}}\right) /(C-1) \times 100
$$

where $N_{n}$ is the number of averages (nine this case), $\bar{L}_{j}$ is the mean activity concentration in the lesion volume of interest (VOI) in slice $j, \bar{B}_{j}$ the mean activity concentration in the background VOI in slice $j$, and $C$ the true lesion-background ratio. Lesion VOIs were delineated based on their known location and size. Background VOIs were delineated as shown in Fig. 6(d). The COV is defined as follows:

$$
\operatorname{Cov}(\%)=\frac{1}{N_{n}} \sum_{j=1}^{N_{n}} \frac{\sigma_{B_{j}}}{\bar{B}_{j}} \times 100
$$

where $\sigma_{B_{j}}$ is the pixel-to-pixel percent standard deviation $(\% \mathrm{SD})$ in the background region of the slice considered.

We also included the error bars (plus and minus the standard deviation over the nine $\mathrm{CRC}$ values).

\section{RESULTS}

\section{A. Analytical optimization}

Figure 5(a) shows the result of the optimization process: the volume sensitivity is plotted as a function of the collimator radius and for different detector radii. The volume sensitivity is maximized for a collimator radius of $150.2 \mathrm{~mm}$ and a detector radius of $172.67 \mathrm{~mm}$, which corresponds to four rings of 34 dSiPMs. For these parameters, 24 phs with an opening angle of $95^{\circ}$ can be placed in one ring without having any overlap between the pinhole projections [Fig. 5(b)]. The pinhole aperture diameter needed to achieve the target resolution of $7.5 \mathrm{~mm}$ is $0.62 \mathrm{~mm}$. We used eight rings of 24 pinholes for sufficient axial sampling. The rings are rotated relative to one another to improve angular sampling. The first ring has pinholes at $0^{\circ}$, $15^{\circ}, 30^{\circ}, 45^{\circ}, 60^{\circ}, \ldots$ The second ring has pinholes at $7.5^{\circ}$, $22.5^{\circ}, 37.5^{\circ}, 52.5^{\circ}, \ldots$ The third ring has again pinholes at $0^{\circ}, 15^{\circ}, 30^{\circ}, 45^{\circ}, 60^{\circ}, \ldots$.

Although the 24-pinhole setup has the highest sensitivity, we wanted to compare the system with another setup having more pinholes and thus better angular sampling. Therefore, we selected a second setup with more pinholes per ring at the cost of a slightly decreased sensitivity. The second setup has the same detector radius of $172.67 \mathrm{~mm}$ but a collimator radius of $156.63 \mathrm{~mm}$ so that 34 pinholes with an opening angle of $92.6^{\circ}$ can be placed in one ring without having any overlap between the pinhole projections [Fig. 5(b)]. The pinhole aperture diameter needed to achieve the target resolution of $7.5 \mathrm{~mm}$ is $0.34 \mathrm{~mm}$. Again, we use eight rings of pinholes for sufficient axial sampling. The first ring has pinholes at $0^{\circ}, 10.59^{\circ}, 21.18^{\circ}$, $31.76^{\circ}, 42.35^{\circ}, \ldots$ The second ring has pinholes at $5.29^{\circ}$, $15.88^{\circ}, 26.47^{\circ}, 37.06^{\circ}, \ldots$ The third ring has again pinholes at $0^{\circ}, 10.59^{\circ}, 21.18^{\circ}, 31.76^{\circ}, 42.35^{\circ}, \ldots$.

The volume sensitivities of the 24- and 34-pinhole systems are 395 and $345 \mathrm{cps} / \mathrm{MBq}$, respectively.

\section{B. Noiseless phantom studies}

\section{B.1. Spatial resolution}

In the cold-rod phantom, the 24-pinhole setup [Fig. 6(b)] performs better than the 34-pinhole setup [Fig. 6(c)]. With the 24 pinholes, we can distinguish the $5 \mathrm{~mm}$ cold rods. The 34-pinhole setup shows a very good resolution at the edge of the phantom but suffers from sampling issues in the center, which can be explained by the gaps between the detectors. In the 24-pinhole setup, the gaps between the 34 detectors are back projected irregularly, i.e., to different areas in image space. In the 34-pinhole setup, however, in four out of the eight pinhole rings, the gaps between the 34 detectors are always back projected to the center. For this reason, the central region
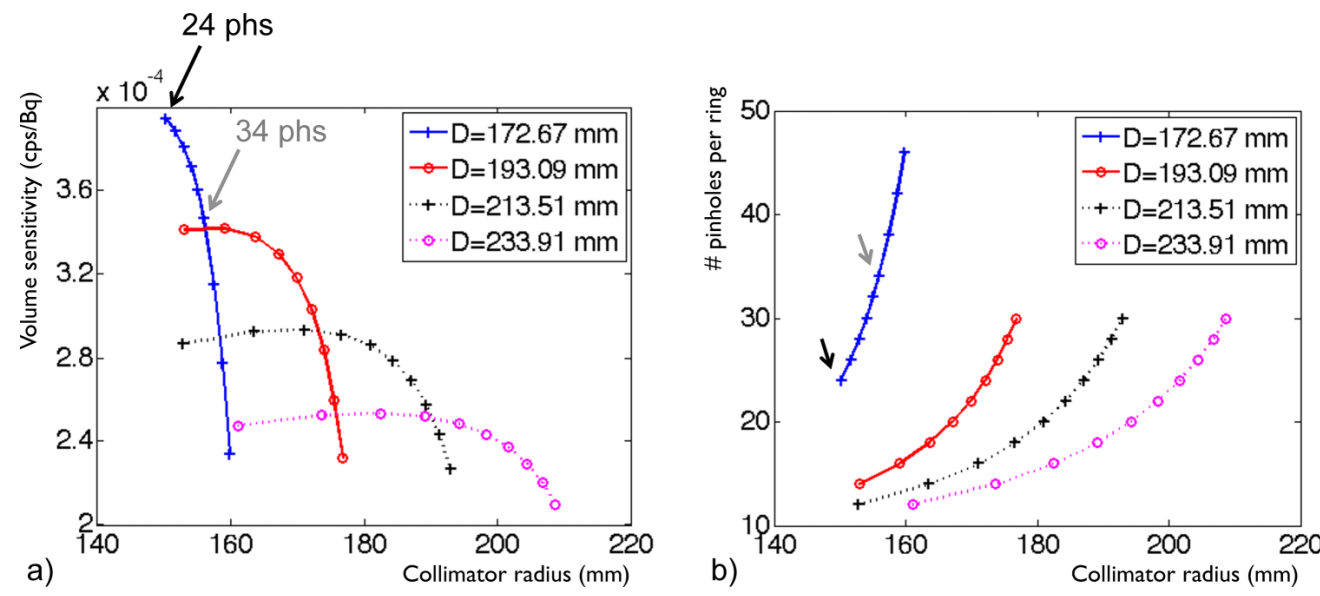

Fig. 5. (a) Volume sensitivity as a function of the collimator and detector radius. (b) Number of pinholes that can be placed in one ring on the collimator, given a certain collimator and detector radius. The points on the curves represent various configurations of different number of pinholes. The arrows indicate which points of the curve correspond to the 24-pinhole and 34-pinhole setup used during the simulations. 

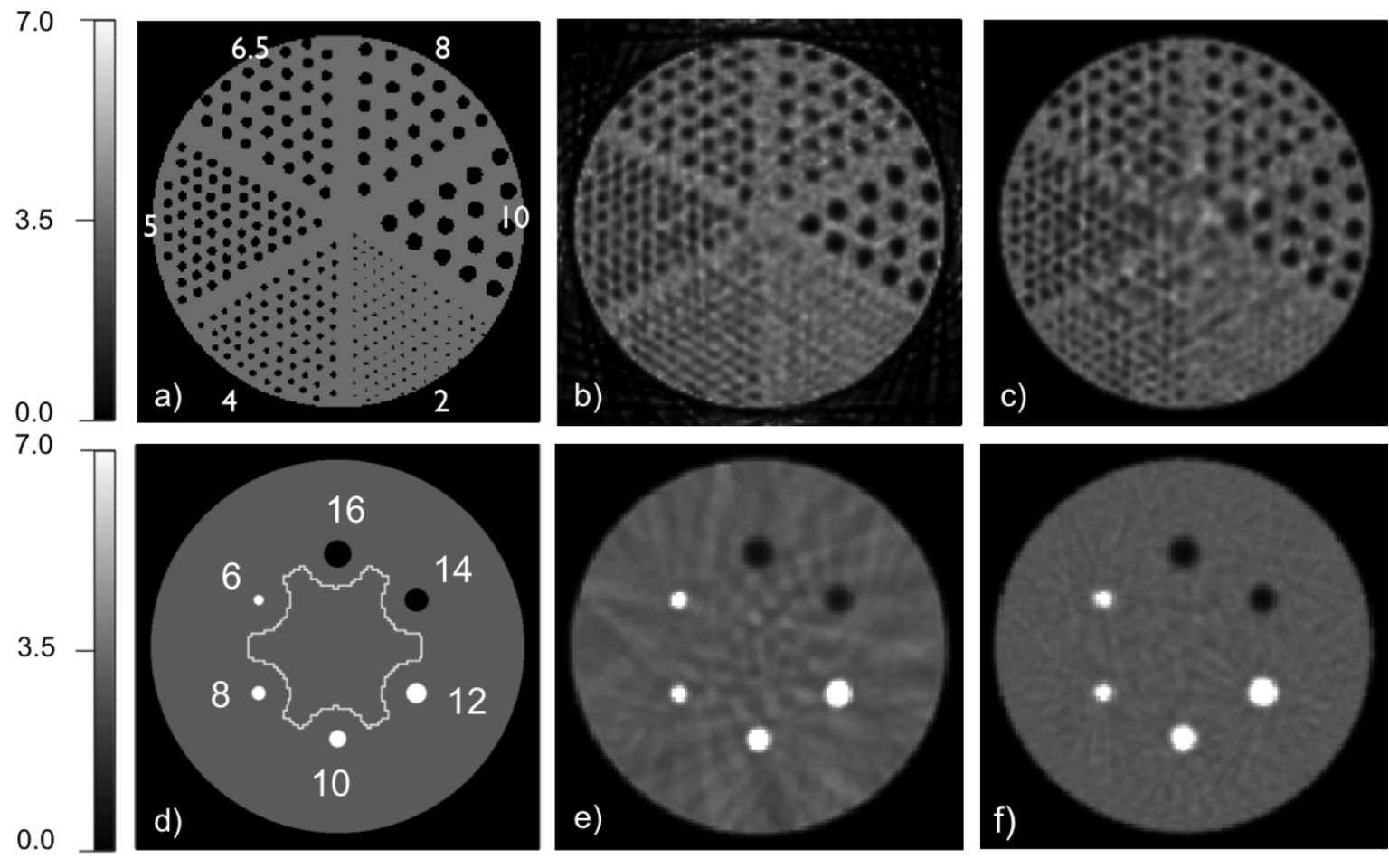

Fig. 6. Noiseless images of the multipinhole systems after 600 MLEM iterations. (a) Transverse view of the cold-rod phantom. (b) Cold-rod phantom with $8 \times 24$ pinholes. (c) Cold-rod phantom with $8 \times 34$ pinholes. (d) Transverse view of the contrast phantom with a white line indicating where the COV and background activity were evaluated. All hot rods have an uptake ratio of 7:1. (e) Contrast phantom $(8 \times 24 \mathrm{phs})$. (f) Contrast phantom $(8 \times 34 \mathrm{phs})$.

is not sufficiently sampled and cannot be reconstructed without artifacts.

\section{B.2. Angular sampling}

On the other hand, the 34 pinholes clearly perform better in the contrast phantom [Figs. 6(c) and 6(f)]. The contrast phantom has a large uniform region in the center, which is typically easier to reconstruct. The reconstruction of this phantom suffers less from the gaps and benefits more from the increased angular sampling.

\section{B.3. Axial sampling}

The individual disks of the Defrise phantom are well resolved for both the 24- and 34-pinhole setup, which shows that axial sampling completeness is achieved in both setups [Figs. 7(b), 7(c), 7(e), and 7(f)].

\section{C. Comparison with a clinical triple-head UHR fan beam system}

\section{C.1. CNR}

Figure 8 shows the Monte Carlo simulated contrast phantoms for both the 24-pinhole multipinhole system and the UHR fan beam system. The images are shown at equal noise level $(\mathrm{COV}=30 \%)$ after a 3D Gaussian filter was applied with a FWHM of $6 \mathrm{~mm}$ and a kernel size of $25 \times 25 \times 25$ voxels. This corresponds to 170 iterations for the MR insert and 471 iterations for the fan beam system. We observe that the noise is less correlated with the UHR FAN system than with the multipinhole system, but we do not observe any visual differences in contrast. For more quantitative results, we also evaluated the contrast-to-noise ratio (Fig. 9). We see that at $30 \%$ COV the CRC is $16 \%$ and $25 \%$ lower with the 24 pinholes in the 8 and $10 \mathrm{~mm}$ hot lesions, respectively, but in the $6 \mathrm{~mm}$ hot and $16 \mathrm{~mm}$ cold lesions the CRC is $13 \%$ and $11 \%$ higher, respectively, with the 24-pinhole setup. In the other lesions, the difference is smaller than the standard deviation of the CRC. We also observe a larger standard deviation in the cold lesions when acquired with the multipinhole system. This is due to interslice differences, which are bigger in a multipinhole system than in a fan beam system, which has the same sensitivity profile in every slice.

\section{C.2. Hoffman phantom}

Figure 10 shows the reconstructed images of the Hoffman phantom after postsmoothing with a 3D Gaussian filter (FWHM of $7.5 \mathrm{~mm}$ and kernel size of $25 \times 25 \times 25$ voxels). The images are shown at equal noise level $(\mathrm{COV}=30 \%)$, with the $\mathrm{COV}$ calculated in uniform parts of the phantom (the thalamus and the cerebellum). This corresponds to 164 iterations for the MR insert and 453 iterations for the fan beam system. The cold spot is slightly more visible in the multipinhole image than in the fan beam image and on the line profile we observe a higher peak-to-valley ratio in the multipinhole image. However, this result is based on a single noise realization and its only purpose is to illustrate the feasibility of SPECT/MRI in a more realistic phantom. For a quantitative comparison of image quality, we refer to the CNR study in Sec. 3.C.1. 

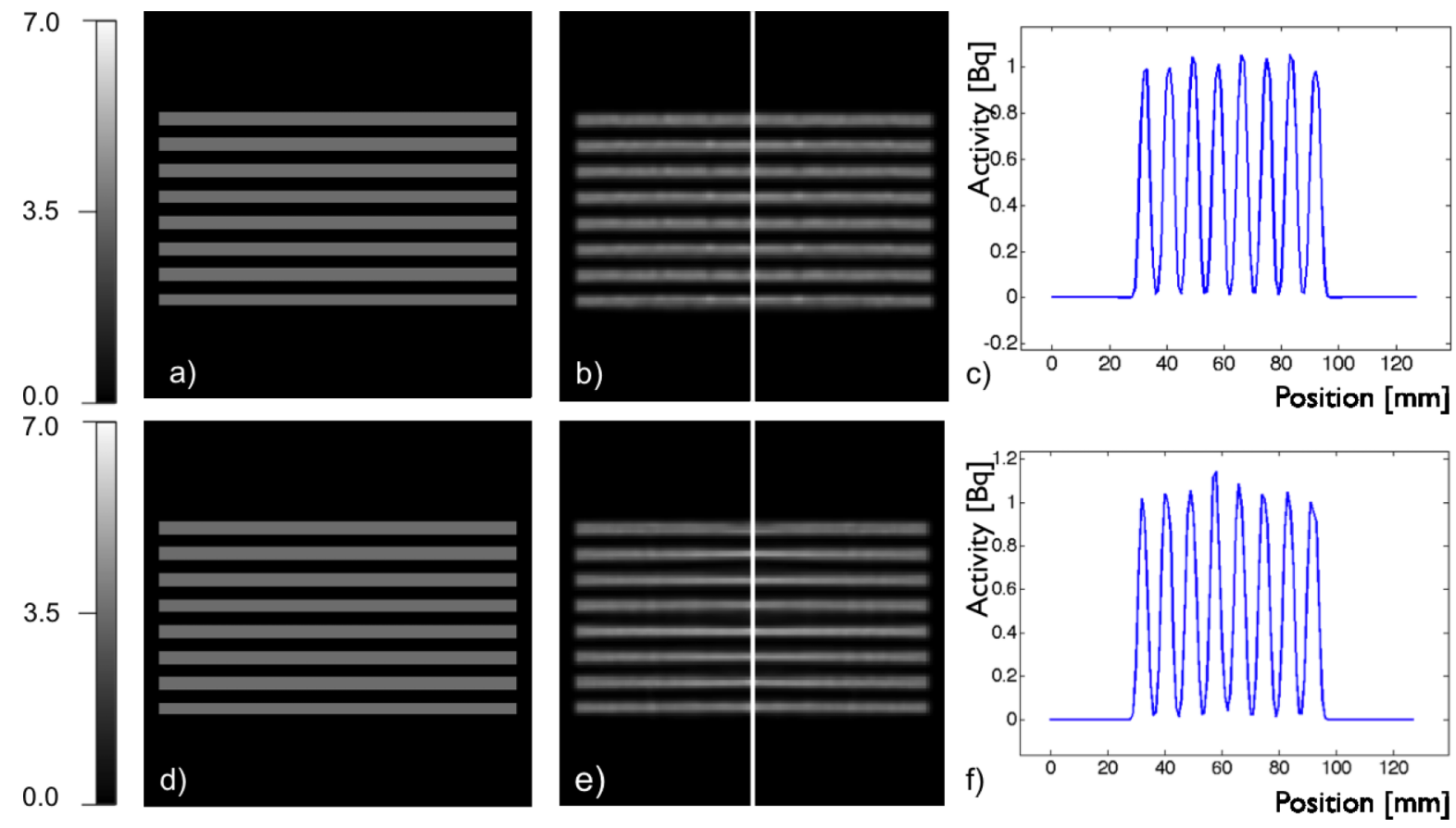

FIG. 7. Noiseless images of the Defrise phantom after 600 MLEM iterations. (a) Coronal view of the Defrise phantom. (b) Noiseless simulation of the Defrise phantom $(8 \times 24$ phs). (c) Axial line profile of the noiseless simulation $(8 \times 24$ phs). (d) Coronal view of the Defrise phantom. (e) Noiseless simulation of the Defrise phantom $(8 \times 34 \mathrm{phs})$. (f) Axial line profile of the noiseless simulation $(8 \times 34 \mathrm{phs})$.

\section{DISCUSSION}

Figure 5(a) depicts the result of the optimization study, which shows that the sensitivity is generally higher for a detector with a smaller radius (and thus longer axial length) compared to a detector with a larger radius (and thus a shorter axial length). For a fixed collimator radius, a smaller detector radius corresponds to more minification and consequently more pinhole projections can be used to fill the detector area, resulting in increased sensitivity. However, when there is too much minification, the sensitivity drops fast (as we can see in the last points of the curve corresponding to $D=172.67)$. The reason for this drop is that at high minification, the pinhole apertures need to be extremely small in order to achieve the target resolution, which results in reduced sensitivity. We found that volume sensitivity is maximized for a detector radius of $172.67 \mathrm{~mm}$, which corresponds to four rings of 34 detector modules. Further decreasing the detector radius would result in such a high degree of minification that the target resolution of $7.2 \mathrm{~mm}$ can no longer be achieved.

Figure 5(a) also shows that the highest volume sensitivity is obtained with a collimator radius of $150.2 \mathrm{~mm}$. Further reducing the collimator radius is not possible as the Tx/Rx MR coil still needs to fit within it.

We performed noiseless simulations with two different setups. The first setup with $8 \times 24$ pinholes and four rings of 34 detectors is the one with the highest sensitivity. The second setup with $8 \times 34$ pinholes and four rings of 34 detectors has more angular projections but it experiences some sampling issues due to the gaps between the detectors. Therefore, we decided to continue with the 24-pinhole setup.

When we compare the 24 pinholes and UHR FAN system in terms of contrast-to-noise ratio, we see that the multipinhole system performs better in the $6 \mathrm{~mm}$ hot and $16 \mathrm{~mm}$ cold lesions and worse in the $8 \mathrm{~mm}$ hot and $10 \mathrm{~mm}$ hot lesions. In the other
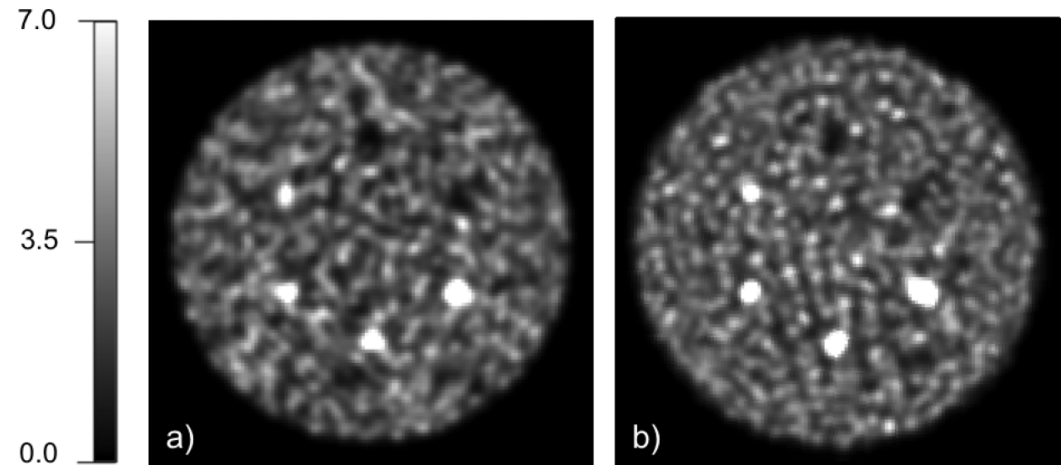

FIG. 8. Monte Carlo simulations of a contrast phantom at equal noise (COV 30\%) with (a) $8 \times 24$ pinholes, 170 iterations. (b) UHR fan beam, 471 iterations. 

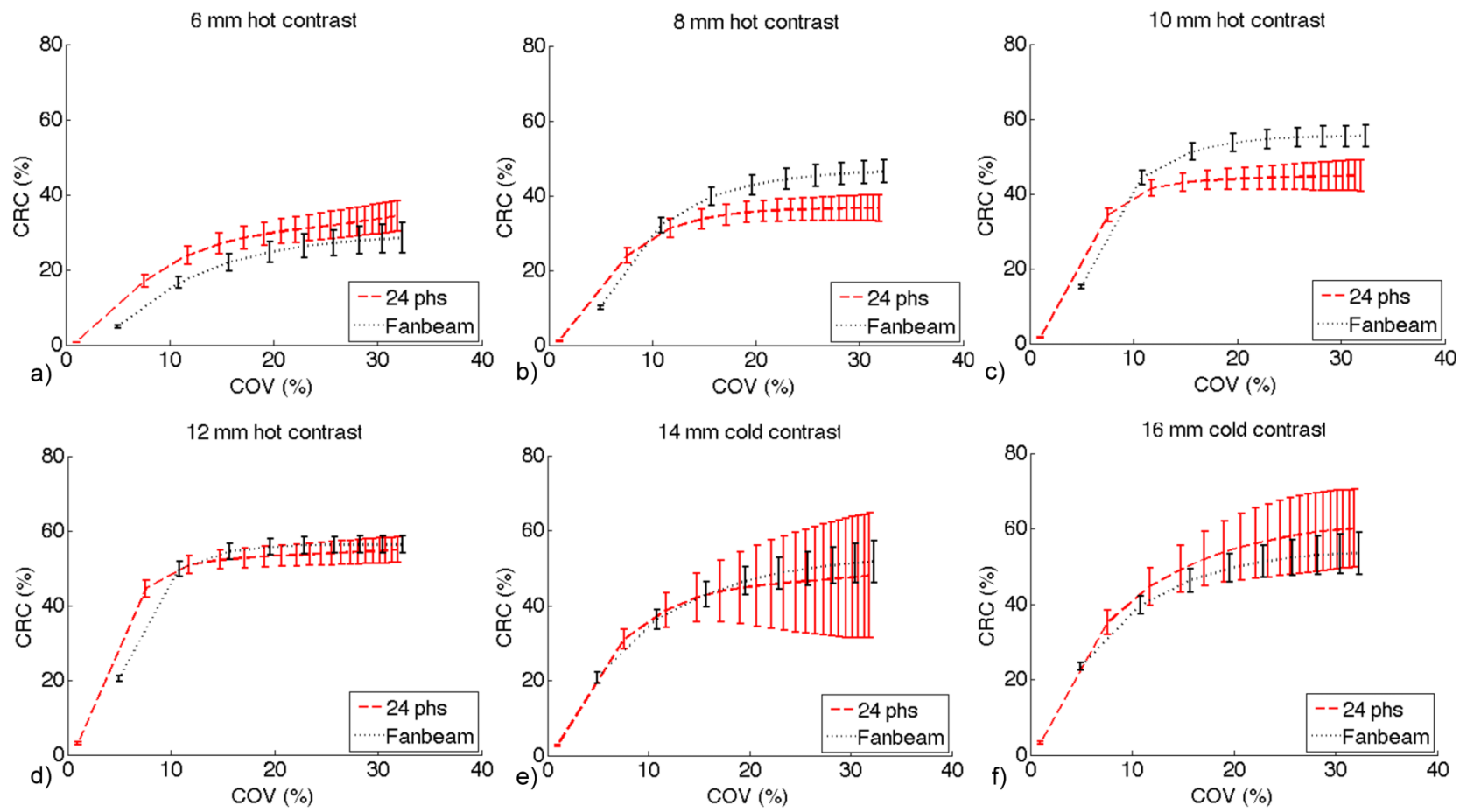

FIG. 9. Contrast-to-noise plots with error bars (plus and minus the standard deviation) for both the multipinhole systems (gray) and the UHR fan beam collimator (black): (a) $6 \mathrm{~mm}$ hot lesion, (b) $8 \mathrm{~mm}$ hot lesion, (c) $10 \mathrm{~mm}$ hot lesion, (d) $12 \mathrm{~mm}$ hot lesion, (e) $14 \mathrm{~mm}$ cold lesion, (f) $16 \mathrm{~mm}$ cold lesion.

lesions, the difference is smaller than the standard deviation of the measured CRC. We also compared both systems based on a visual evaluation of a Hoffman phantom simulated with a clinically realistic activity and scan time and found that the $9 \mathrm{~mm}$ cold lesion was slightly better visible on the multipinhole image. Although this visual evaluation of the Hoffman phantom was based on a single noise realization and is not strong enough to be conclusive, it gives us the confidence that brain SPECT/MRI is feasible with a multipinhole insert so that in the future we can build the system and continue with measured data and a task-based comparison (e.g., lesion detectability).
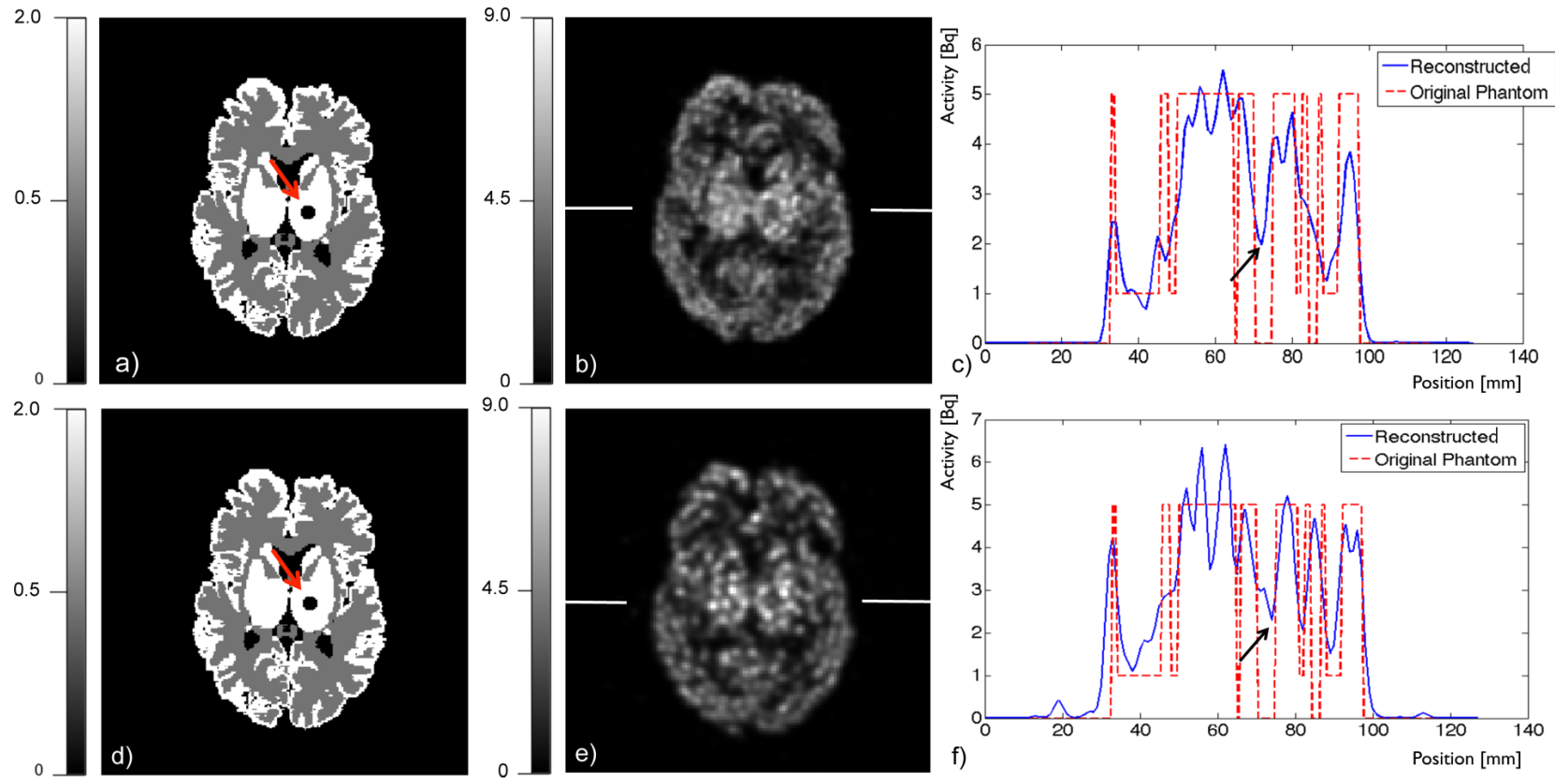

FIG. 10. Monte Carlo simulations of a Hoffman phantom with the multipinhole setup $(8 \times 24$ pinholes $)$ and the UHR fan beam setup, compared at equal noise level (COV 30\%). (a) Transverse view of the phantom with a $9 \mathrm{~mm}$ cold lesion. (b) Monte Carlo simulation with the multipinhole setup, 164 iterations. (c) Line profile for the multipinhole setup. (d) Transverse view of the phantom with a $9 \mathrm{~mm}$ cold lesion. (e) Monte Carlo simulation with the UHR fan beam system, 453 iterations. (f) Line profile for the UHR fan beam system. 


\section{CONCLUSION}

We have optimized a stationary brain SPECT insert for MRI based on high-resolution dSiPMs and a full-ring multipinhole collimator and found that the optimal system consists of four rings of 34 detector modules and a cylindrical collimator with a radius of $150.2 \mathrm{~mm}$ and eight rings of 24 pinholes with an aperture of $0.62 \mathrm{~mm}$ diameter. Analytical calculations predict a system spatial resolution of $7.2 \mathrm{~mm}$ in the center of the FOV and a volume sensitivity of $395 \mathrm{cps} / \mathrm{MBq}$. This volume sensitivity is lower than a triple-head system with fan beam collimators of the same resolution, but higher than a dual-head system.

We first performed noiseless simulations of a Defrise and a cold-rod phantom and demonstrated sufficient axial sampling and a reconstructed resolution high enough to resolve rods as small as $5 \mathrm{~mm}$. Moreover, Monte Carlo simulations of a contrast phantom demonstrated a contrast-to-noise ratio comparable to that of a triple-head fan beam system. Finally, we reconstructed noisy projection data of a Hoffman phantom with a $9 \mathrm{~mm}$ cold lesion to illustrate the application of the SPECT/MR insert in a realistic human brain phantom.

In conclusion, these results show that brain SPECT/MRI is feasible with a multipinhole insert and that we can expect image quality to be similar to the best clinical systems available.

\section{ACKNOWLEDGMENTS}

This work was performed at Ghent University and supported by iMinds Medical IT and the 7th Framework Programme of the European Union through the SUBLIMA project (Grant Agreement No. 241711). Roel Van Holen is supported by a fellowship of the Research Foundation Flanders, Karen Van Audenhaege is supported by a doctoral fellowship of the agency for Innovation by Science and Technology (IWT, Belgium), and Christian Vanhove is supported by the GROUPID consortium of Ghent University.

\footnotetext{
${ }^{a)}$ Author to whom correspondence should be addressed. Electronic mail: roel.vanholen@ugent.be

${ }^{1}$ V. Keereman, Y. Fierens, T. Broux, Y. De Deene, M. Lonneux, and S. Vandenberghe, "MRI-based attenuation correction for PET/MRI using ultrashort echo time sequences," J. Nucl. Med. 51, 812-818 (2010).

${ }^{2}$ D. Strul, D. Cash, S. Keevil, P. Halsted, S. Williams, and P. Marsden, "Gamma shielding materials for MR-compatible PET," IEEE Trans. Nucl. Sci. 50, 60-69 (2003).

${ }^{3}$ D. Strul, D. Cash, S. Keevil, P. Halstead, S. Williams, and P. Marsden, "MR-compatible shields for $511 \mathrm{keV}$ gamma photons: A feasibility study,' in 2000 IEEE Nuclear Science Symposium. Conference Record (Cat. No.00CH37149) (IEEE, San Diego, CA, 2001), Vol. 3, pp. 16/11-16/15. ${ }^{4}$ D. Renker, "New trends on photodetectors," Nucl. Instrum. Methods Phys. Res., Sect. A: Accel., Spectrom., Detect. Assoc. Equip. 571, 1-6 (2007).

${ }^{5}$ I. Britvitch, I. Johnson, D. Renker, A. Stoykov, and E. Lorenz, "Characterisation of Geiger-mode avalanche photodiodes for medical imaging applications," Nucl. Instrum. Methods Phys. Res., Sect. A: Accel., Spectrom., Detect. Assoc. Equip. 571, 308-311 (2007).

${ }^{6} \mathrm{E}$. Roncali and S. R. Cherry, "Application of silicon photomultipliers to positron emission tomography," Ann. Biomed. Eng. 39, 1358-1377 (2011). ${ }^{7}$ J. Wehner, B. Weissler, P. Dueppenbecker, P. Gebhardt, D. Schug, W. Ruetten, F. Kiessling, and V. Schulz, "PET/MRI insert using digital SiPMs:
}

Investigation of MR-compatibility," Nucl. Instrum. Methods Phys. Res., Sect. A: Accel., Spectrom., Detect. Assoc. Equip. 734, 116-121 (2014).

${ }^{8} \mathrm{~S}$. Vandenberghe and P. K. Marsden, "PET-MRI: A review of challenges and solutions in the development of integrated multimodality imaging," Phys. Med. Biol. 60, R115-R154 (2015).

${ }^{9}$ A. Kolb, H. F. Wehrl, M. Hofmann, M. S. Judenhofer, L. Eriksson, R. Ladebeck, M. P. Lichy, L. Byars, C. Michel, H. P. Schlemmer, M. Schmand, C. D. Claussen, V. Sossi, and B. J. Pichler, "Technical performance evaluation of a human brain PET/MRI system,” Eur. Radiol. 22, 1776-1788 (2012).

${ }^{10}$ P. Veit-Haibach, F. P. Kuhn, F. Wiesinger, G. Delso, and G. Von Schulthess, "PET-MR imaging using a tri-modality PET/CT-MR system with a dedicated shuttle in clinical routine," Magn. Reson. Mater. Phys., Biol. Med. 26, 25-35 (2013).

${ }^{11}$ J. J. Griesmer, J. Futey, N. Ojha, and M. Morich, "Whole-body PET-MR imaging system initial calibration results," in IEEE Nuclear Science Symposium Conference Record (IEEE, Knoxville, TN, 2010), pp. 2174-2176.

${ }^{12}$ G. Delso and S. Ziegler, “PET/MRI system design,” Eur. J. Nucl. Med. Mol. Imaging 36, 86-92 (2009).

${ }^{13}$ C. Catana, Y. Wu, M. S. Judenhofer, J. Qi, B. J. Pichler, and S. R. Cherry, "Simultaneous acquisition of multislice PET and MR images: Initial results with a MR-compatible PET scanner," J. Nucl. Med. 47, 1968-1976 (2006).

${ }^{14}$ M. S. Judenhofer, C. Catana, B. K. Swann, S. B. Siegel, W.-I. Jung, R. E. Nutt, S. R. Cherry, C. D. Claussen, and B. J. Pichler, "PET/MR images acquired with a compact MR-compatible PET detector in a 7-T magnet," Radiology 244, 807-814 (2007).

${ }^{15}$ V. Schulz, T. Solf, B. Weissler, P. Gebhardt, P. Fischer, M. Ritzert, V. Mlotok, C. Piemonte, N. Zorzi, M. Melchiorri, S. Vandenberghe, V. Keereman, T. Schaeffter, and P. K. Marsden, "A preclinical PET/MR insert for a human 3T MR scanner," in IEEE Nuclear Science Symposium Conference Record (IEEE, Orlando, FL, 2009), pp. 2577-2579.

${ }^{16}$ B. Weissler, P. Gebhardt, P. Diippenbecker, A. Salomon, D. Schug, C. Lerche, D. Wirtz, W. Renz, K. Schumacher, B. Zwaans, P. Marsden, F. Kiessling, and V. Schulz, "Design concept of world's first preclinical PET/MR insert with fully digital silicon photomultiplier technology," in IEEE Nuclear Science Symposium and Medical Imaging Conference Record (IEEE, Anaheim, CA, 2012), pp. 2113-2116.

${ }^{17}$ M. J. Hamamura, S. Ha, W. W. Roeck, L. T. Muftuler, D. J. Wagenaar, D. Meier, B. E. Patt, and O. Nalcioglu, "Development of an MR-compatible SPECT system (MRSPECT) for simultaneous data acquisition," Phys. Med. Biol. 55, 1563-1575 (2010).

${ }^{18}$ S. Azman, J. Gjaerum, D. Meier, L. Muftuler, G. Maehlum, O. Nalcioglu, B. E. Patt, B. Sundal, M. Szawlowski, B. M. Tsui, and D. J. Wagenaar, "A nuclear radiation detector system with integrated readout for SPECT/MR small animal imaging," in 2007 IEEE Nuclear Science Symposium Conference Record (IEEE, Honolulu, HI, 2007), pp. 2311-2317.

${ }^{19}$ D. Meier, D. J. Wagenaar, S. Chen, J. Xu, J. Yu, and B. M. W. Tsui, "A SPECT camera for combined MRI and SPECT for small animals," Nucl. Instrum. Methods Phys. Res., Sect. A: Accel., Spectrom., Detect. Assoc. Equip. 652, 731-734 (2011).

${ }^{20}$ D. Meier, D. J. Wagenaar, G. Mæhlum, B. Sundal, B. E. Patt, S. Chen, J. Xu, B. M. W. Tsui, M. Hamamura, S. Ha, W. W. Roeck, and O. Nalcioglu, "Development of pre-clinical SPECT for MRI," IFMBE Proc. 25, 798-801 (2009).

${ }^{21}$ L.-J. Meng, J.-W. Tan, and G. Fu, "Design study of an MRI compatible ultra-high resolution SPECT for in vivo mice brain imaging," in 2007 IEEE Nuclear Science Symposium Conference Record (IEEE, Honolulu, HI, 2007), pp. 2956-2960.

${ }^{22}$ J.-W. Tan, L. Cai, and L.-J. Meng, "A prototype of the MRI-compatible ultra-high resolution SPECT for in vivo mice brain imaging," in 2009 IEEE Nuclear Science Symposium Conference Record (NSS/MIC) (IEEE, Orlando, FL, 2009), Vol. 61801, pp. 2800-2805.

${ }^{23}$ L. Cai, X. Lai, Z. Shen, C.-T. Chen, and L.-J. Meng, "MRC-SPECT: A sub$500 \mu \mathrm{m}$ resolution MR-compatible SPECT system for simultaneous dualmodality study of small animals," Nucl. Instrum. Methods Phys. Res. 734, 147-151 (2014)

${ }^{24}$ P. Busca, C. Fiorini, A. D. Butt, M. Occhipinti, R. Peloso, R. Quaglia, F. Schembari, P. Trigilio, G. Nemeth, P. Major, K. Erlandsson, and B. F. Hutton, "Simulation of the expected performance of INSERT: A new multi-modality SPECT/MRI system for preclinical and clinical imaging," Nucl. Instrum. Methods Phys. Res. 734, 141-146 (2014).

${ }^{25}$ Mediso Medical Imaging Systems (available URL: http://www.mediso. com/products.php?fid=2,11\&pid=83). Accessed June 2014. 
${ }^{26}$ C. Bouckaert, S. Vandenberghe, and R. V. Holen, "Evaluation of a compact, high-resolution SPECT detector based on digital silicon photomultipliers,' Phys. Med. Biol. 59, 7521-7539 (2014).

${ }^{27}$ M. Rogulski, H. Barber, H. Barrett, R. Shoemaker, and J. Woolfenden, "Ultra-high-resolution brain SPECT imaging: Simulation results," IEEE Trans. Nucl. Sci. 40, 1123-1129 (1993).

${ }^{28}$ R. Van Holen, B. Vandeghinste, K. Deprez, and S. Vandenberghe, "Design and performance of a compact and stationary microSPECT system," Med Phys. 40, 112501 (11pp.) (2013).

${ }^{29}$ K. Deprez, S. Vandenberghe, K. Van Audenhaege, and R. Van Holen, "Rapid additive manufacturing of MR compatible multi-pinhole collimators with selective laser melting of tungsten powder," Med. Phys. 40, 012501 (11pp.) (2013).

${ }^{30}$ A. M. Samoudi, K. Van Audenhaege, G. Vermeeren, M. Poole, E. Tanghe, L. Martens, R. Van Holen, and W. Joseph, "Analysis of eddy currents induced by transverse and longitudinal gradient coils in different tungsten collimators geometries for SPECT/MRI integration," Magn. Reson. Med. (published online 2014).

${ }^{31}$ M. C. Goorden, M. C. M. Rentmeester, and F. J. Beekman, "Theoretical analysis of full-ring multi-pinhole brain SPECT," Phys. Med. Biol. 54, 6593-6610 (2009).

${ }^{32}$ K. Van Audenhaege, S. Vandenberghe, K. Deprez, B. Vandeghinste, and R. Van Holen, "Design and simulation of a full-ring multi-lofthole collimator for brain SPECT," Phys. Med. Biol. 58, 6317-6336 (2013).

${ }^{33}$ H. O. Anger, M. R. Powell, D. C. van Dyke, L. R. Schaer, R. Fawwaz, and Y. Yano, "Recent applications of the scintillation camera," Strahlentherapie 65, 70-93 (1967).

${ }^{34} \mathrm{R}$. Accorsi and S. D. Metzler, "Analytic determination of the resolutionequivalent effective diameter of a pinhole collimator," IEEE Trans. Med. Imaging 23, 750-763 (2004).
${ }^{35} \mathrm{P}$. M. Joseph, "An improved algorithm for reprojecting rays through pixel images," IEEE Trans. Med. Imaging 1, 192-196 (1982).

${ }^{36}$ B. Vandeghinste, S. Member, C. Vanhove, J. D. Beenhouwer, and R. V. Holen, "Absolute quantification in multi-pinhole micro-SPECT for different isotopes," in 2011 IEEE Nuclear Science Symposium Conference Record (NSS/MIC) (IEEE, Valencia, Spain, 2011), pp. 3720-3724.

${ }^{37}$ S. Jan, G. Santin, D. Strul, S. Staelens, K. Assié, D. Autret, D. Avner, R. Barbier, M. Bardiès, P. Bloomfield, D. Brasse, V. Breton, P. Bruyndonckx, I. Buvat, A. Chatziioannou, Y. Choi, Y. Chung, C. Comtat, D. Donnarieix, L. Ferrer, S. Glick, C. Groiselle, S. Kerhoas-Cavata, A. Kirov, V. Kohli, M. Koole, M. Krieguer, J. van der Laan, F. Lamare, G. Largeron, C. Lartizien, D. Lazaro, M. Maas, L. Maigne, F. Mayet, F. Melot, S. Nehmeh, E. Pennacchio, J. Perez, U. Pietrzyk, F. Rannou, M. Rey, D. Schaart, R. Schmidtstein, L. Simon, T. Song, J. Vieira, D. Visvikis, R. Van de Walle, C. Wieers, and E. Morel, "GATE-A simulation toolkit for PET and SPECT," Phys. Med. Biol. 49, 1-19 (2004).

${ }^{38}$ H. J. Kim, B. R. Zeeberg, F. H. Fahey, A. N. Bice, E. J. Hoffman, and R. C. Reba, "Three-dimensional SPECT simulations of a complex three-dimensional mathematical brain model and measurements of the three-dimensional physical brain phantom,” J. Nucl. Med. 32, 1923-1930 (1991).

${ }^{39}$ C. Vanhove, A. Andreyev, M. Defrise, J. Nuyts, and A. Bossuyt, "Resolution recovery in pinhole SPECT based on multi-ray projections: A phantom study," Eur. J. Nucl. Med. Mol. Imaging 34, 170-180 (2007).

${ }^{40}$ D. Ruijters, B. M. ter Haar Romeny, and P. Suetens, "Efficient GPU-based texture interpolation using uniform B-splines," J. Graphics, GPU, Game Tools 13, 61-69 (2008).

${ }^{41}$ R. A. Moyer, "A low-energy multihole converging collimator compared with a pinhole collimator," J. Nucl. Med. 15, 59-64 (1973). 